\title{
Correction to: Nonuniform reinjection probability density function in type $V$ intermittency
}

\author{
Sergio Elaskar • Ezequiel del Río • \\ L. Gutierrez Marcantoni
}

Published online: 4 May 2018

(C) Springer Science+Business Media B.V., part of Springer Nature 2018

Correction to: Nonlinear Dyn (2018) 92:683-697

https://doi.org/10.1007/s11071-018-4083-7

The information regarding the first author's affiliations was incomplete in the original publication. It is correctly shown here.

The original article can be found online at https://doi.org/10. 1007/s11071-018-4083-7.

S. Elaskar $(\varangle) \cdot$ L. Gutierrez Marcantoni

Departamento de Aeronáutica, FCEFyN, Instituto de Estudios Avanzados en Ingeniería y Tecnología, CONICET and Universidad Nacional de Córdoba, Córdoba, Argentina e-mail: sergio.elaskar@gmail.com; selaskar@unc.edu.ar

E. del Río · S. Elaskar

Departamento de Física Aplicada, ETSIAE, Universidad

Politécnica de Madrid, Madrid, Spain

e-mail: ezequiel.delrio@upm.es 